\title{
Improvement of Indigofera zollingeriana Production and Methionine Content through Inoculation of Nitrogen-Fixing Bacteria
}

\author{
P. S. Hutapea ${ }^{a, *}$, L. Abdullaha, ${ }^{a}$, P. D. M. H. Karti ${ }^{a}$, \& I. Anas ${ }^{b}$ \\ ${ }^{a}$ Department of Nutrition and Feed Technology, Faculty of Animal Science, Bogor Agricultural University \\ ${ }^{b}$ Faculty of Agriculture, Bogor Agricultural University \\ Kampus IPB Darmaga, Bogor 16680, Indonesia \\ *Email of corresponding author: lukiabdullah@gmail.com; priskila.hutapea@gmail.com \\ (Received 24-11-2017; Reviewed 21-12-2017; Accepted 25-01-2018)
}

\begin{abstract}
Indigofera zollingeriana is a tremendous potential as a forage plant due to its high protein content. This experiment was conducted to increase production and nutrient quality of I. zollingeriana biomass, especially methionine content through inoculation of nitrogen fixing bacteria. Nine isolates obtained from nodule of I. zollingeriana were screened for 37 days in greenhouse, resulting in three desirable isolates. These three selected isolates at density of $10^{5} \mathrm{cfu} / \mathrm{mL}$ and $10^{7} \mathrm{cfu} / \mathrm{mL}$ were inoculated as much as $1 \mathrm{~mL}$ per seedling. Uninoculated plants were also grown as control treatment, i.e., positive $\left(+0.05 \% \mathrm{KNO}_{3}(\mathrm{w} / \mathrm{v})\right)$ and negative. The experiment was laid out in a completely randomized design with four replicates and carried out for three months. Plant biomass was analyzed for amino acid and other nutrient contents. Moreover, nodulation activity was also observed by counting and weighting the nodules. The results showed that Bradyrhizobium sp. P8 828, Bradyrhizobium sp. PZS_A08, and Roseomonas sp. CMS4Y-2-2 significantly increased shoot production. Subsequent experiment showed that plant inoculation with Bradyrhizobium sp. PZS_A08 at $10^{5} \mathrm{cfu} / \mathrm{mL}$ significantly increased dry matter content. Inoculation with Bradyrhizobium sp. PZS_A08 and Roseomonas sp. CMS4Y-2-2 at 10 ${ }^{7} \mathrm{cfu} / \mathrm{mL}$ produced higher crude protein contents that were better than the application of nitrogen fertilizer. Inoculation treatment with Bradyrhizobium sp. P8 828 at $10^{5} \mathrm{cfu} / \mathrm{mL}$ was the most effective in improving methionine content. It is concluded that production and quality of biomass obtained from inoculation treatments were comparable to the application of inorganic N-fertilizer.
\end{abstract}

Keywords: Indigofera zollingeriana, nitrogen fixing bacteria, inoculation, methionine, Bradyrhizobium, Roseomonas

\section{INTRODUCTION}

The availability of feed raw material is a crucial factor to support feed self-sufficiency. Due to the lack of national production, some protein-based feed materials such as grains, fish meal, and other materials have been dependent on import. Recently, green concentrate has already considered to fulfill nutrient requirement in feed. Besides high crude protein, the concentrate also contains vitamin, mineral, digestible fiber, antioxidant properties, and bioactive compounds.

Indigofera zollingeriana is a legume plant which is considered as one of the potential forages for both monogastrics and ruminants animals. Suharlina et al. (2016) stated that this plant was capable of producing high crude protein biomass and also had a higher digestibility compared to other legumes. This legume contained high crude protein (29.76\%-29.83\%) and a low tannin $(0.09 \%-0.65 \%)$ (Abdullah, 2010). It was also rich in digestible protein $(75 \%-87 \%)$, utilisable fiber including Neutral Detergent Fiber (49\%-57\%) and Acid Detergent Fiber (32\%-38\%), and showed a high dry mat- ter digestibility (72\%-81\%) (Abdullah \& Suharlina, 2010). Supplementation of $I$. zollingeriana to dairy goat feed increased feed conversion, dry matter digestibility (from $17 \%$ to $73 \%$ ), feed efficiency $(8 \%-17 \%)$, protein utilization efficiency (from $1 \%$ to $2.5 \%$ ), average daily milk production (from 121 to $383 \mathrm{~mL} / \mathrm{d}$ ), and decreased methane emission as well as feed production cost as much as US\$0.39 (Abdullah et al., 2012; Suharlina et al., 2016). The addition of $I$. zollingeriana flour for feed supplementation of laying quail replaced $50 \%$ use of soybean meal, enabling to increase feed consumption, egg weight, yolk colour, cholesterol properties, and to decrease malondhyaldehide content (Faradillah et al., 2015).

Due to the potential benefit, further research must be conducted. Inoculation with nitrogen-fixing bacteria is one of effective way. Bacteria provide the plant with fixed $\mathrm{N}_{2}$, then get oxygen and net photosynthate in return. Inoculation with spesific bacteria obtained from I. zollingeriana nodule was expected to improve biomass production and quality. The improvement of crude protein especially amino acid composition were highlighted in this study. 
Currently, feed formulation based on amino acid has become a great concern than crude protein. Then, amino acid composition is being considered as an important requirement of qualified protein-based feed materials. Methionine and lysine are limiting amino acids in most of plant-based feed and often become limiting amino acids in milk production. Therefore, methionine and lysine are often applied in feed supplementation for both ruminant and monogastric feeds. Methionine (2 $\mathrm{kg} /$ day) and lysine (1.5 kg/day) supplementations into dairy cow could increase milk production (Wang et al., 2010). Methionine supplementation into low-protein poultry ration also increased feed efficiency, protein synthesis, and decreased fat synthesis (Bunchasak, 2009).

Amino acid composition in animal-based feed material is more balance than the plant-based feed material. Lysine and tryptophan are the most limiting amino acids in cereals, whereas methionine is limited in leguminose. Many researchers, genetically improved amino acid content (Ufaz \& Galili, 2008). The aim of this experiment was to increase the production of I. zollingeriana biomass and enhance its crude protein percentage, especially amino acid composition of $I$. zollingeriana through inoculation of nitrogen-fixing bacteria.

\section{MATERIALS AND METHODS}

\section{Collection of Indigofera zollingeriana Nodules}

Nodules were obtained from 3-months old $I$. zollingeriana grown at 3 locations, i.e Education and Research Field of Bogor Agricultural University (IPB) in Jonggol, goat breeding farm in Cilumbang, and Agrostology Laboratory (Faculty of Animal Science, Bogor Agricultural University). Nodules were separated from the roots and cleaned from adhering soil, and then preserved in plastic tube filled with silica gel.

\section{Isolation and Maintenance of Isolates}

Isolation process was conducted at Soil Biotechnology Laboratory, Faculty of Agriculture IPB. Nodules were washed under running tap water, and then the surfaces were sterilized by soaking them in a sterilized distilled water (10 s) and 5.25\% NaClO (20 s). Thereafter, the nodules were rinsed 3 times using sterilized distilled water each for $10 \mathrm{~s}$. Furthermore, they were soaked in alcohol $95 \%$ for $10 \mathrm{~s}$ and then rinsed 2 times using sterilized distilled water. The sterilized nodules were soaked in sterile sodium chloride solution and crushed. One loopful nodule juice was streaked on Yeast Extract Mannitol Agar (YEMA) and incubated for 3-5 d at room temperature (Somasegaran and Hoben, 1985, Graham \& Parker, 1964).

Isolate morphology was observed based on the color and shape of bacterial colonies. Isolate denoted different colors and shapes were separated into different YEMAs. Purification was done by subculturing single colony repeatedly. The isolates were preserved in refigerator at $4^{\circ} \mathrm{C}$ for further use.

\section{Screening and Identification of Isolates}

I. zollingeriana seeds were soaked in the water to obtain good quality seeds. Scarification was done to facilitate imbibision, considering that I. zollingeriana had a hard seed pericarp. Prior to planting, the seeds were sterilized by soaking them in alcohol 95\% (10 s) and then in $\mathrm{NaClO} 5.25 \%$ (about 3-5 min). Then, they were rinsed in sterilized distilled water (6 times), and soaked in sterilized distilled water at $70^{\circ} \mathrm{C}(2 \mathrm{~h})$.

The sterilized seeds (10 seeds) were planted in glass pot containing sterilized sands (sterilized using autoclave for $30 \mathrm{~min}$ ). The seeds were inoculated with 5 -d old isolates grown in YEM broth. Two healthy seedlings in each pot were grown for $37 \mathrm{~d}$ and watered every $2 \mathrm{~d}$ with sterilized distilled water. The most effective isolates were then identified using 16s r-RNA. Strain identification was conducted at Indonesian Center for Biodiversity and Biotechnology (ICBB).

\section{Greenhouse Experiment and Nutrient Analysis}

The soil used in the experiment was air dried, sieved, and weighed $5 \mathrm{~kg} /$ polybag. Thereafter, the soil was autoclaved for $30 \mathrm{~min}$. The seeds were sterilized by soaking in alcohol 95\% (for $10 \mathrm{~s}$ ) and $5.25 \% \mathrm{NaClO}$ (for $3 \mathrm{~min}$ ). The sterilized seeds were rinsed using sterilized distilled water at least 6 times. Lastly, the seeds were soaked in sterilized distilled water at $70^{\circ} \mathrm{C}$ for $2 \mathrm{~h}$. Ten seeds were sown per polybag. Two healthy seedlings were maintained per polybag.

For inoculation treatment, each seedling was inoculated with $1 \mathrm{~mL}$ isolates, according to treatments. Seedlings were incubated for a week, then a healthy seedling was maintained per polybag. Positive control treatments were uninoculated plant with an addition of $0.05 \% \mathrm{KNO}_{3}(\mathrm{w} / \mathrm{v})$ solution with a dose of $300 \mathrm{~kg} \mathrm{~N} /$ ha. All plants were watered using boiled cool water and sterilized Jensen solution (without sucrose). Jensen solution consisted of $1 \mathrm{~g} \mathrm{~K}_{2} \mathrm{HPO}_{4^{\prime}} 0.5 \mathrm{~g} \mathrm{MgSO}_{4} .7 \mathrm{H}_{2} 0,0.5$ $\mathrm{g} \mathrm{NaCl}, 0.1 \mathrm{~g} \mathrm{FeSO}_{4^{\prime}} 0.005 \mathrm{~g}$ sodium molybdate, and $2 \mathrm{~g}$ $\mathrm{CaCO}$

\section{Experimental Design and Observation}

For isolates screening test, 9 isolates were collected. Treatments consisted of plants inoculated with 9 different isolates and uninoculated plant. The parameters observed included morphological characteristics of bacterial colonies, vertical height, and leaf number (observed once a week), and dry weight of shoot, root, and nodule (determined at the end of experimental period). Nodulation effectivity was determined to investigate the most specific isolate and calculated as biomass dry weight per gram of nodules.

Subsequent experiment consisted of 8 treatments: $\mathrm{R}_{0}$ (negative control); $\mathrm{R}_{1}$ (positive control); $\mathrm{R}_{2} \mathrm{P}_{1}$ (Bradyrhizobium sp. P8 828 isolate at $10^{5} \mathrm{cfu} / \mathrm{mL}$ ); $\mathrm{R}_{2} \mathrm{P}_{2}$ (Bradyrhizobium sp. P8 828 isolate at $10^{7} \mathrm{cfu} / \mathrm{mL}$ ); $\mathrm{R}_{3} \mathrm{P}_{1}$ (Bradyrhizobium sp. PZS_A08 isolate at $10^{5} \mathrm{cfu} / \mathrm{mL}$ ); $\mathrm{R}_{3} \mathrm{P}_{2}$ (Bradyrhizobium sp. PZS_A08 isolate at $10^{7} \mathrm{cfu} / \mathrm{mL}$ ); 
$\mathrm{R}_{4} \mathrm{P}_{1}$ (Roseomonas sp. CMS4Y-2-2 isolate at $10^{5} \mathrm{cfu} / \mathrm{mL}$ ); $\mathrm{R}_{4} \mathrm{P}_{2}$ (Roseomonas sp. CMS4Y-2-2 isolate at $10^{7} \mathrm{cfu} / \mathrm{mL}$ ). All treatments were carried out at 4 replicates, and the observed parameters included nodule number, nodule dry weight, leaf number, vertical height, biomass dry weight, proximate analysis, and amino acid content. The experimental design was completely randomized design. The collected data were then evaluated using analysis of variance (ANOVA), while the significant differences among means were compared using Duncan test at $\mathrm{P}<0.05$ (Steel \& Torrie, 1993).

\section{RESULTS}

\section{Isolate Screening on Indigofera zollingeriana}

All isolates obtained from I. zollingeriana root nodules induced better nodulation than the uninoculated plant. The treatments exhibited significant differences on vertical height, shoot dry weight, and root dry weight (Table 1). JM 1 (Jonggol Merah 1) isolate increased growth and production of I. zollingeriana. The isolate produced higher shoot dry weight and root dry weight by $139.4 \%$ and $115.1 \%$, respectively, compared to uninoculated plant by increasing nodules dry weight as much as $148.1 \%$. JP 1 (Jonggol Putih 1) isolate also exhibited a high productivity after Bradyrhizobium sp. strain PZS_A08. It increased shoot dry weight as much as $64.1 \%$ by increasing nodules dry weight by $153.2 \%$.

Compatibility of isolate-plant could be represented by the effectivity of nodulation, which showed the capability of isolate to produce a high biomass per gram of nodules (Table 2). $\mathrm{JM}_{1}$ isolate was determined to posses a high compatibility because its capability in producing a high shoot and root dry biomass per gram of nodules. It produced $4.68 \mathrm{~g}$ of root and $15.31 \mathrm{~g}$ of shoot per gram of nodules. BM (Cilumbang Merah) isolate was also considered to be compatible with I. zollingeriana because it also produced a high shoot biomass.

The results showed that $\mathrm{JM}_{1}, \mathrm{JP}_{1}$, and $\mathrm{BM}$ isolates (Figure 1) were able to raise biomass production which was supported by a high nodulation activity. Isolate identification revealed that $\mathrm{JM}_{1}$ isolate was attributed to Bradyrhizobium sp. strain PZS_A08, while JP ${ }_{1}$ and BM isolates were attributed to Bradyrhizobium sp. strain P8 828 and Roseomonas sp. strain CMS4Y-2-2, respectively. The latter strain was non-rhizobia bacteria found to be able to form nodules and increase plant growth.

Roseomonas sp. is not common as a Plant Growth Promotor Rhizobacteria. Some researches found that this bacteria were commonly isolated from the blood of patient with cancer (Dé et al., 2004). However, another experiment reported that the bacteria also could be found in seed and root of Phaseolus vulgaris (LopézLopéz et al. 2010). Cohen et al. (2004) found the same sequence in Azospirillum brasilense, Roseomonas fauriae, and Roseomonas genomospesies 6 at 16S-rRNA, 23S-rRNAgenes, ITS1-region, as well as at nifH- and rpOB-genes.

\section{Effect of Inoculation on Indigofera zollingeriana Production}

There was no significant differences in nodulation activity as the result of inoculation with different isolates and population. Roseomonas sp. CMS4Y-2-2 and

Table 2. Nodulation effectivity of Indigofera zollingeriana

\begin{tabular}{ccc}
\hline \multirow{2}{*}{$\begin{array}{c}\text { Code of } \\
\text { isolates }\end{array}$} & \multicolumn{2}{c}{ Variables } \\
\cline { 2 - 3 } & $\begin{array}{c}\text { Nodulation effectivity } \\
\text { on root biomass }\left(\mathrm{g} \mathrm{g}^{-1}\right)\end{array}$ & $\begin{array}{c}\text { Nodulation effectivity } \\
\text { on shoot biomass }\left(\mathrm{g} \mathrm{g}^{-1}\right)\end{array}$ \\
\hline No isolate & $4.79 \pm 1.86$ & $14.70 \pm 3.10$ \\
JL & $2.52 \pm 0.18$ & $8.34 \pm 2.26$ \\
JM1 & $4.68 \pm 1.74$ & $15.31 \pm 4.23$ \\
JM2 & $4.23 \pm 1.55$ & $12.73 \pm 1.60$ \\
JM3 & $2.89 \pm 1.05$ & $10.96 \pm 2.54$ \\
BM & $4.38 \pm 2.33$ & $14.24 \pm 3.52$ \\
BP & $4.31 \pm 2.67$ & $13.66 \pm 4.24$ \\
KF & $4.08 \pm 0.52$ & $10.11 \pm 3.64$ \\
JP1 & $4.54 \pm 1.84$ & $10.19 \pm 2.46$ \\
JP2 & $5.48 \pm 1.00$ & $12.52 \pm 1.87$ \\
\hline
\end{tabular}

Note: nodulation effectivity is shoot or root biomass per gram nodule.

Table 1. Growth and production of Indigofera zollingeriana affected by inoculation of various isolates

\begin{tabular}{cccccc}
\hline \multirow{2}{*}{ Code of isolates } & \multicolumn{5}{c}{ Variables } \\
\cline { 2 - 6 } & $\begin{array}{c}\text { Leaf number } \\
\text { (leaf/plant) }\end{array}$ & $\begin{array}{c}\text { Vertical height } \\
(\mathrm{cm} / \text { plant })\end{array}$ & $\begin{array}{c}\text { NDW } \\
(\mathrm{mg} / \text { plant })\end{array}$ & $\begin{array}{c}\text { SDW } \\
(\mathrm{mg} / \text { plant })\end{array}$ & $\begin{array}{c}\text { RDW } \\
(\mathrm{mg} / \text { plant})\end{array}$ \\
\hline No isolate & $2.89 \pm 0.08$ & $4.33 \pm 0.47^{\mathrm{b}}$ & $2.33 \pm 0.49$ & $31.30 \pm 1.92^{\mathrm{b}}$ & $10.80 \pm 3.72^{\mathrm{c}}$ \\
JL & $3.22 \pm 0.42$ & $4.73 \pm 0.27^{\mathrm{b}}$ & $5.62 \pm 0.65$ & $44.27 \pm 9.65^{\mathrm{b}}$ & $14.37 \pm 0.93^{\mathrm{bc}}$ \\
JM1 & $4.06 \pm 0.75$ & $6.18 \pm 0.75^{\mathrm{a}}$ & $5.78 \pm 1.83$ & $74.93 \pm 8.12^{\mathrm{a}}$ & $23.23 \pm 5.61^{\mathrm{a}}$ \\
JM2 & $3.17 \pm 0.49$ & $5.51 \pm 0.21^{\mathrm{ab}}$ & $3.50 \pm 1.17$ & $43.07 \pm 11.07^{\mathrm{b}}$ & $13.15 \pm 1.72^{\mathrm{bc}}$ \\
JM3 & $3.06 \pm 0.31$ & $4.54 \pm 0.59^{\mathrm{b}}$ & $3.40 \pm 1.78$ & $37.63 \pm 5.87^{\mathrm{b}}$ & $10.47 \pm 1.18^{\mathrm{c}}$ \\
BM & $2.78 \pm 0.98$ & $4.67 \pm 0.60^{\mathrm{b}}$ & $2.58 \pm 0.99$ & $36.03 \pm 5.19^{\mathrm{b}}$ & $11.72 \pm 2.02^{\mathrm{c}}$ \\
BP & $3.33 \pm 0.27$ & $4.26 \pm 0.39^{\mathrm{b}}$ & $4.05 \pm 1.42$ & $44.13 \pm 6.10^{\mathrm{b}}$ & $13.32 \pm 5.15^{\mathrm{bc}}$ \\
KF & $3.28 \pm 0.28$ & $5.03 \pm 0.06^{\mathrm{ab}}$ & $4.22 \pm 1.04$ & $37.58 \pm 9.71^{\mathrm{b}}$ & $16.45 \pm 3.93^{\mathrm{ab}}$ \\
JP1 & $3.00 \pm 0.24$ & $5.31 \pm 0.80^{\mathrm{ab}}$ & $5.90 \pm 3.07$ & $51.37 \pm 17.26^{\mathrm{b}}$ & $21.00 \pm 3.65^{\mathrm{ab}}$ \\
JP2 & $3.11 \pm 0.21$ & $5.16 \pm 0.61^{\mathrm{ab}}$ & $4.83 \pm 0.51$ & $50.15 \pm 2.69^{\mathrm{b}}$ & $20.93 \pm 2.11^{\mathrm{ab}}$ \\
\hline
\end{tabular}

Note: Means with different superscripts differ significantly at vertical height $(\mathrm{P}<0.05)$, shoot dry weight and root dry weight $(\mathrm{P}<0.01)$. NDW= nodule dry weight; SDW= shoot dry weight; RDW= root dry weight. 


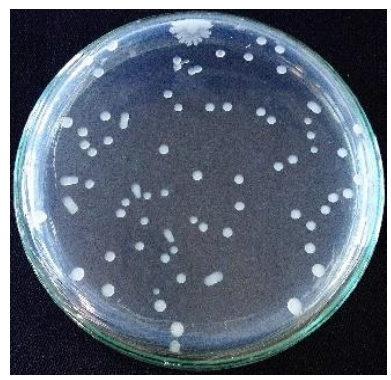

A

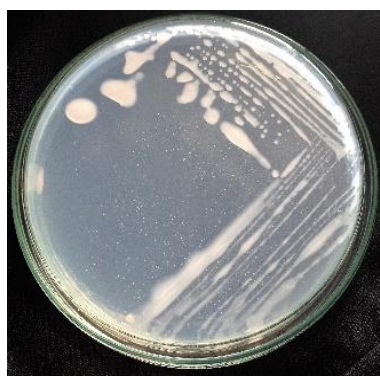

B

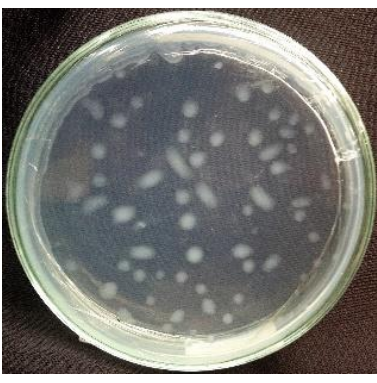

C

Figure 1. Selected isolates: Bradyrhizobium sp. strain P8 828 (A); Roseomonas sp. strain CMS4Y- 2-2 (B); Bradyrhizobium sp. strain PZS_ A08 (C)

Bradyrhizobium sp. PZS_A08 had a high compatibility with I. zollingeriana. Both isolates were more effective in producing biomass per nodule dry weight compared to Bradyrhizobium sp. P8 828. This result was consistent with the result found in screening experiment.

Negative control treatment showed the least plant growth and biomass production. Production and nodulation activity of the plant with inoculation treatment tended to be higher than positive and negative control despite the nonsignificant difference (Table 3). Roseomonas sp. CMS4Y-2-2, Bradyrhizobium sp. PZS_A08, and Bradyrhizobium sp. P8 828 produced higher biomass by $29.9 \%, 27.9 \%$, and $24 \%$, respectively, compared to plant in negative control treatment at $10^{5} \mathrm{cfu} / \mathrm{mL}$. Inoculation treatment also resulted in a higher biomass production compared to positive control treatment. This is understandable that production of biomass treated by inoculation treatment is comparable, even higher, compared to nitrogen fertilizer at a dose of $300 \mathrm{~kg} \mathrm{~N} / \mathrm{ha}$.

\section{Effect of Inoculation on Nutrient Quality of Indigofera zollingeriana}

Different treatments only gave a significant difference on dry matter percentage. Bradyrhizobium sp. PZS_A08 at $10^{7} \mathrm{cfu} / \mathrm{mL}$ produced the highest dry matter percentage that was $8.56 \%$ higher than negative control treatment. Interestingly, although the increasing of nitrogen availability resulted in higher dry matter, crude fat percentage decreased in this treatment. In the absence of nitrogen fertilizer or nitrogen-fixing bacteria, the crude fat content of plant was higher.

Similar to dry matter percentage, a high availability of nitrogen in fertilizer $\mathrm{KNO}_{3}$ as well as through inoculation increased the percentage of crude protein compared to negative control treatment. The highest crude protein content was obtained at inoculations with Bradyrhizobium sp. PZS_A08 (107 cfu/mL), Roseomonas sp. CMS4Y-2-2 (10 $\mathrm{cfu} / \mathrm{mL})$, and Bradyrhizobium sp. P8 828 $\left(10^{5} \mathrm{cfu} / \mathrm{mL}\right)$. Those isolates, respectively, gave $25.11 \%$, $15.44 \%$, and $10.18 \%$ higher crude protein content compared to control treatment (Table 4).

\section{Effect of Inoculation on Amino Acid Properties of Indigofera zollingeriana}

Application of $\mathrm{KNO}_{3}$ and inoculation of nitrogenfixing bacteria were able to raise essential amino acid content in I. zollingeriana biomass. The comparison between amino acid content in this experiment and other experiments was shown in Table 5. Application of $\mathrm{KNO}_{3}$ fertilizer yielded the highest chemical score

Table 3. Growth and production of Indigofera zollingeriana treated by inoculation of nitrogen fixing bacteria

\begin{tabular}{ccccccc}
\hline & \multicolumn{9}{c}{ Variables } \\
\cline { 2 - 7 } Treatments & $\begin{array}{c}\text { Nodulation } \\
\text { effectivity } \\
(\mathrm{g} / \mathrm{g})\end{array}$ & $\begin{array}{c}\text { Biomass dry } \\
\text { weight } \\
\text { (g/plant) }\end{array}$ & $\begin{array}{c}\text { Nodule dry } \\
\text { weight (g/plant) }\end{array}$ & $\begin{array}{c}\text { Nodule number } \\
\text { (nodule/plant) }\end{array}$ & $\begin{array}{c}\text { Vertical height } \\
\text { (cm/plant) }\end{array}$ & $\begin{array}{c}\text { Leaf number } \\
\text { (leaf/plant) }\end{array}$ \\
\hline R0 & - & $15.74 \pm 3.40$ & $0.83 \pm 0.24$ & $352.67 \pm 39.50$ & $48.69 \pm 3.91$ & $152.83 \pm 47.30$ \\
R1 & - & $17.83 \pm 5.54$ & $0.91 \pm 0.26$ & $386.75 \pm 119.13$ & $50.07 \pm 8.21$ & $135.00 \pm 51.04$ \\
R2P1 & $17.28 \pm 2.86$ & $19.52 \pm 2.93$ & $1.17 \pm 0.37$ & $376.00 \pm 125.90$ & $53.29 \pm 8.08$ & $104.25 \pm 30.75$ \\
R2P2 & $15.10 \pm 1.82$ & $16.96 \pm 3.13$ & $1.13 \pm 0.18$ & $581.25 \pm 119.21$ & $44.13 \pm 8.11$ & $129.25 \pm 56.14$ \\
R3P1 & $16.27 \pm 1.76$ & $20.14 \pm 5.09$ & $1.26 \pm 0.37$ & $543.25 \pm 337.71$ & $45.86 \pm 3.97$ & $109.88 \pm 32.42$ \\
R3P2 & $22.72 \pm 7.76$ & $16.07 \pm 4.50$ & $0.84 \pm 0.57$ & $230.33 \pm 106.08$ & $43.16 \pm 4.00$ & $126.58 \pm 11.88$ \\
R4P1 & $24.44 \pm 2.88$ & $20.44 \pm 4.21$ & $0.84 \pm 0.14$ & $384.50 \pm 32.42$ & $49.22 \pm 11.21$ & $158.31 \pm 59.93$ \\
R4P2 & $19.01 \pm 7.23$ & $17.27 \pm 2.30$ & $1.01 \pm 0.40$ & $467.75 \pm 81.36$ & $52.62 \pm 3.52$ & $119.63 \pm 22.53$ \\
\hline
\end{tabular}

Note: R0 (negative control) with Jensen solution; R1 (positive control) with $300 \mathrm{~kg}$ N/ha + Jensen solution, R2 (inoculation with Bradyrhizobium sp. P8 828); R3 (inoculated with Bradyrhizobium sp. PZS_A08); R4 (inoculated with Roseomonas sp. CMS4Y-2-2); P1 (inoculated with total of 10 ${ }^{5}$ cfu/mL); P2 (inoculated with total of $10^{7} \mathrm{cfu} / \mathrm{mL}$ ). 
Table 4. Nutrient content of Indigofera zollingeriana biomass treated by inoculation of nitrogen fixing bacteria

\begin{tabular}{ccccc}
\hline \multirow{2}{*}{ Treatments } & \multicolumn{3}{c}{ Variables } \\
\cline { 2 - 5 } & $\begin{array}{c}\text { Dry matter } \\
(\%)\end{array}$ & $\begin{array}{c}\text { Crude protein } \\
(\%)\end{array}$ & $\begin{array}{c}\text { Amino acid chemical } \\
\text { score }(\%)\end{array}$ & $\begin{array}{c}\text { Crude fat } \\
(\%)\end{array}$ \\
\hline R0 & $21.26 \pm 0.91^{\mathrm{ab}}$ & $17.68 \pm 1.82$ & $5.57 \pm 2.00$ & $4.93 \pm 0.16$ \\
R1 & $21.91 \pm 1.57^{\mathrm{ab}}$ & $19.14 \pm 2.35$ & $8.26 \pm 2.03$ & $4.61 \pm 0.44$ \\
R2P1 & $20.36 \pm 1.88^{\mathrm{b}}$ & $19.48 \pm 1.22$ & $7.02 \pm 2.19$ & $4.83 \pm 0.27$ \\
R2P2 & $21.45 \pm 0.58^{\mathrm{ab}}$ & $17.44 \pm 2.56$ & $3.60 \pm 2.43$ & $4.40 \pm 0.44$ \\
R3P1 & $23.08 \pm 0.92^{\mathrm{a}}$ & $18.75 \pm 1.14$ & $5.91 \pm 1.98$ & $4.30 \pm 0.27$ \\
R3P2 & $20.24 \pm 0.74^{\mathrm{b}}$ & $22.12 \pm 6.74$ & $6.26 \pm 1.10$ & $4.17 \pm 0.38$ \\
R4P1 & $21.77 \pm 1.33^{\mathrm{ab}}$ & $19.17 \pm 2.22$ & $6.76 \pm 1.65$ & $4.32 \pm 0.47$ \\
R4P2 & $20.96 \pm 0.91^{\mathrm{b}}$ & $20.41 \pm 2.55$ & $6.07 \pm 0.69$ & $4.45 \pm 0.23$ \\
\hline
\end{tabular}

Note: R0 (negative control) with Jensen solution; R1 (positive control) with $300 \mathrm{~kg}$ N/ha + Jensen solution, R2 (inoculation with Bradyrhizobium sp. P8 828); R3 (inoculated with Bradyrhizobium sp. PZS_A08); R4 (inoculated with Roseomonas sp. CMS4Y-2-2); P1 (inoculated with total of 105 cfu/mL); $\mathrm{P} 2$ (inoculated with total of $10^{7} \mathrm{cfu} / \mathrm{mL}$ ). Means in the same column with different superscripts differ significantly $(\mathrm{P}<0.05)$.

Tabel 5. Comparison of essential amino acid content in top leaf and biomass (top and bottom leaf) of I. zollingeriana under various treatments of inoculation

\begin{tabular}{|c|c|c|c|c|c|c|}
\hline \multirow[b]{2}{*}{$\begin{array}{l}\text { Essential } \\
\text { amino acid }\end{array}$} & \multicolumn{6}{|c|}{ Treatments } \\
\hline & $\begin{array}{l}\text { Biomass } \\
\text { (R1) }\end{array}$ & $\begin{array}{l}\text { Biomass } \\
\text { (R2P1) }\end{array}$ & $\begin{array}{l}\text { Biomass } \\
\text { (R4P1) }\end{array}$ & $\begin{array}{l}\text { Biomass } \\
\text { (R3P2) }\end{array}$ & $\begin{array}{c}\text { Biomass } \\
\text { (Foliar fertilizer } \\
\text { treatment) } \\
\text { (Abdullah et al., 2016) }\end{array}$ & $\begin{array}{c}\text { Top leaf } \\
\text { (Palupi et al., 2014) }\end{array}$ \\
\hline Histidine & $0.71 \pm 0.30$ & $0.70 \pm 0.13$ & $0.70 \pm 0.13$ & $0.69 \pm 0.39$ & 0.04 & 0.67 \\
\hline Threonine & $0.50 \pm 0.08$ & $0.54 \pm 0.21$ & $0.56 \pm 0.16$ & $0.39 \pm 0.24$ & 0.06 & 1.14 \\
\hline Arginine & $1.16 \pm 0.14$ & $1.29 \pm 0.44$ & $0.97 \pm 0.26$ & $0.82 \pm 0.52$ & 0.155 & 1.67 \\
\hline Methionine & $0.43 \pm 0.08$ & $0.54 \pm 0.23$ & $0.49 \pm 0.24$ & $0.27 \pm 0.14$ & 0.045 & 0.43 \\
\hline Valine & $0.83 \pm 0.12$ & $0.86 \pm 0.16$ & $0.70 \pm 0.28$ & $0.51 \pm 0.30$ & 0.13 & 1.56 \\
\hline Phenilalanin & $0.75 \pm 0.07$ & $0.71 \pm 0.20$ & $0.53 \pm 0.17$ & $0.47 \pm 0.28$ & - & 1.6 \\
\hline Isoleucin & $0.68 \pm 0.18$ & $0.58 \pm 0.19$ & $0.55 \pm 0.12$ & $0.45 \pm 0.26$ & 0.045 & 1.35 \\
\hline Leucine & $1.10 \pm 0.07$ & $1.28 \pm 0.24$ & $1.32 \pm 0.44$ & $0.98 \pm 0.71$ & 0.13 & 2.26 \\
\hline Lysine & $0.81 \pm 0.11$ & $0.85 \pm 0.15$ & $0.92 \pm 0.21$ & $0.62 \pm 0.38$ & 0.04 & 1.57 \\
\hline $\begin{array}{l}\text { Chemical score } \\
\text { of AA }\end{array}$ & 8.27 & 7.02 & 6.76 & 6.26 & 0.5 & 10.4 \\
\hline
\end{tabular}

Note: R1 (positive control) with $300 \mathrm{~kg}$ N/ha + Jensen solution, R2 (inoculation with Bradyrhizobium sp. P8 828); R3 (inoculated with Bradyrhizobium sp. PZS_A08); R4 (inoculated with Roseomonas sp. CMS4Y-2-2); P1 (inoculated with total of $10^{5} \mathrm{cfu} / \mathrm{mL}$ ); P2 (inoculated with total of $10^{7} \mathrm{cfu} / \mathrm{mL}$ ); AA= amino acid.

of amino acid content present in biomass, followed by Bradyrhizobium sp. P8 $828\left(10^{5} \mathrm{cfu} / \mathrm{mL}\right)$, Roseomonas sp. CMS4Y-2-2 (105 cfu/mL), and Bradyrhizobium sp. PZS_A08 $\left(10^{7} \mathrm{cfu} / \mathrm{mL}\right)$. Negative control treatment had the lowest chemical score of amino acid content.

In this study, methionine and histidine present in I. zollingeriana biomass was regarded high as in top leaf part, even higher. Inoculation with Bradyrhizobium sp. P8 828 at $10^{5} \mathrm{cfu} / \mathrm{mL}$ yielded biomass with methionine content of $0.54 \%$ while methionine content in top leaf analysed by Palupi et al. (2014) was $0.43 \%$. In addition, methionine content was 12 times higher, compared to methionine content in foliar treatment found at previous experiment (Abdullah et al., 2016). Lysine content of this experiment was still lower than in top leaf, but 22 times higher than in foliar treatment. Nitrogen availability through $\mathrm{KNO}_{3}$ addition and nitrogen-fixing bacteria inoculation were apparently more effective compared to foliar treatment.
Non essential amino acid content in I. zollingeriana biomass was found to be higher than in other experiments (foliar treatment) (Abdullah et al., 2016). Proline increased significantly among all non essential amino acid, followed by tyrosine and aspartate. The increases in proline, tyrosine, and aspartate were $12.5 \%, 10.3 \%$, and $9.14 \%$, respectively, compared to the experiment of Abdullah et al. (2016) (Table 6). The improved threonine, lysine, methionine, and isoleucine percentages may be associated with the increase in aspartate percentage.

\section{DISCUSSION}

\section{Isolate Specificity on Indigofera zollingeriana}

All isolates which were inoculated at screening process were obtained from the same nodules of the plant, but only three isolates were proven to be effective in increasing plant biomass. Zhang et al. (2014) found that 
Tabel 6. Comparison of non essential amino acid content in top leaf and biomass (top and bottom leaf) of I. zollingeriana under various treatments of inoculation

\begin{tabular}{lccccccccc}
\hline & \multicolumn{10}{c}{ Treatments } \\
\cline { 2 - 10 } $\begin{array}{l}\text { Nonesensial } \\
\text { amino acid }\end{array}$ & $\begin{array}{c}\text { Biomass } \\
\text { (R0) }\end{array}$ & $\begin{array}{c}\text { Biomass } \\
\text { (R1) }\end{array}$ & $\begin{array}{c}\text { Biomass } \\
\text { (R2P1) }\end{array}$ & $\begin{array}{c}\text { Biomass } \\
\text { (R2P2) }\end{array}$ & $\begin{array}{c}\text { Biomass } \\
\text { (R3P1) }\end{array}$ & $\begin{array}{c}\text { Biomass } \\
\text { (R3P2) }\end{array}$ & $\begin{array}{c}\text { Biomass } \\
\text { (R4P1) }\end{array}$ & $\begin{array}{c}\text { Biomass } \\
\text { (R4P2) }\end{array}$ & $\begin{array}{c}\text { Biomass } \\
\text { (foliar fertilizer }) \\
\text { (Abdullah } \text { et al., } \\
\text { 2016) }\end{array}$ \\
\hline Cystein & $0.34 \pm 0.13$ & $0.48 \pm 0.11$ & $0.43 \pm 0.09$ & $0.29 \pm 0.30$ & $0.29 \pm 0.03$ & $0.26 \pm 0.15$ & $0.36 \pm 0.15$ & $0.50 \pm 0.27$ & - \\
Tyrosine & $0.38 \pm 0.11$ & $0.77 \pm 0.28$ & $0.72 \pm 0.20$ & $0.46 \pm 0.21$ & $0.57 \pm 0.15$ & $0.38 \pm 0.32$ & $0.67 \pm 0.27$ & $0.88 \pm 0.26$ & 0.085 \\
Proline & $0.68 \pm 0.31$ & $0.94 \pm 0.18$ & $0.93 \pm 0.41$ & $0.46 \pm 0.34$ & $0.66 \pm 0.15$ & $0.60 \pm 0.53$ & $0.69 \pm 0.43$ & $0.71 \pm 0.62$ & 0.075 \\
Alanine & $0.53 \pm 0.43$ & $0.54 \pm 0.43$ & $0.35 \pm 0.18$ & $0.48 \pm 0.34$ & $0.28 \pm 0.01$ & $0.42 \pm 0.29$ & $0.52 \pm 0.17$ & $0.41 \pm 0.10$ & 0.08 \\
Glysine & $0.36 \pm 0.26$ & $0.36 \pm 0.02$ & $0.35 \pm 0.04$ & $0.26 \pm 0.23$ & $0.30 \pm 0.09$ & $0.26 \pm 0.15$ & $0.31 \pm 0.09$ & $0.45 \pm 0.30$ & 0.075 \\
Serine & $0.41 \pm 0.17$ & $0.46 \pm 0.19$ & $0.59 \pm 0.15$ & $0.32 \pm 0.17$ & $0.38 \pm 0.04$ & $0.38 \pm 0.25$ & $0.50 \pm 0.11$ & $0.62 \pm 0.28$ & 0.16 \\
Glutamate & $2.78 \pm 0.57$ & $2.85 \pm 0.88$ & $2.63 \pm 1.52$ & $1.79 \pm 0.74$ & $1.95 \pm 0.66$ & $1.98 \pm 1.39$ & $2.35 \pm 0.90$ & $2.66 \pm 1.50$ & 0.36 \\
Arpartate & $1.20 \pm 0.21$ & $1.55 \pm 0.28$ & $1.33 \pm 0.53$ & $1.01 \pm 0.57$ & $1.14 \pm 0.38$ & $0.95 \pm 0.65$ & $1.09 \pm 0.24$ & $1.42 \pm 0.36$ & 0.17 \\
\hline
\end{tabular}

Note: R0 (negative control) with Jensen solution; R1 (positive control) with $300 \mathrm{~kg}$ N/ha + Jensen solution, R2 (inoculation with Bradyrhizobium sp. P8 828); R3 (inoculated with Bradyrhizobium sp. PZS_A08); R4 (inoculated with Roseomonas sp. CMS4Y-2-2); P1 (inoculated with total of 105 cfu/mL); P2 (inoculated with total of $10^{7} \mathrm{cfu} / \mathrm{mL}$ ).

Mesorhizobium ciceri USDA 3378T was more competitive than Mezorhizobium muleiense and Mesorhizobium mediterraneum despite all of them were isolated from the same plant.

Through screening process, Bradyrhizobium sp. strain PZS_A08, Bradyrhizobium sp. strain P8 828, and Roseomonas sp. CMS4Y-2-2 were able to produce the highest biomass production compared to the other isolates, whereas only Bradyrhizobium sp. strain PZS_A08 and Roseomonas sp. CMS4Y-2-2 could effectively produce biomass per gram nodule. Further experiment found that both isolates were effective in producing a high biomass per gram nodule and crude protein content. Bradyrhizobium sp. strain P8 828 was more effective in increasing amino acid (both essential and non essential) as well as amino acid chemical score.

The isolates not only showed symbiotic activity with I. zollingeriana, but were also capable of competing with other bacteria. Nodulation activity was still found in uninoculated plant regardless of sterilization process, which could be linked with the activity of native nodulating-strain bacteria in the soil. Surprisingly, production and nutrient quality of inoculated plant were higher than that of negative control, and comparable with positive control treatment (some parameters were higher). High production and nutrient quality were caused by the capability of isolate in competing with native bacteria.

In symbiotic relationship, both plant host and bacteria attained beneficial effects to each other as seen between Rhizobium sp. BARIRGm901 and soybean (Alam et al. 2015). Symbiotic relationship between rhizobia and plant host is divided into mutualistic and parasitic. Both rhizobia could form nodules, but parasitic rhizobia could only fix a little $\mathrm{N}_{2}$ or even none. It was a great loss for the host plant because nodule formation consumes a lot of energy.

Some legumes could impose sanction to deal with that problem. Legume would restrict net photosynthesis as well as oxygen allocation to rhizobia to inhibit cell reproduction of rhizobia. The reduction of bacteria population would decrease nodulation activity, so the plant could keep the photosynthate for their growths and productions. If the host plant could not distinguish between the effective strain and ineffective strain, the ineffective one would still receive enough photosynthate (Frisien \& Mathias, 2009).

Rhizobia activity on fixing nitrogen defines the action of host plant in allocating net photoynthate. Kiers et al. (2006) stated that the host plant would allocate oxygen and photosynthate at the rate of fixed nitrogen. Medicago truntula is a plant that can impose sanction to ineffective strain (Rangin et al., 2010). Through this experiment, I. zollingeriana was assumed to be able to impose sanction to ineffective strain. The ineffective native strain might get less net photosynthate and oxygen allocation, whereas the chosen isolates received enough amounts.

Among all isolates, isolate obtained from Jonggol showed the best symbiotic capability. The soil in Jonggol was dryer than those in the other locations, which made the bacteria more competitive. Therefore, although isolates were obtained from similar plant species, bacteria strain and their characteristics might be different. Soil characteristic at different sites affected microbial growth and characteristics (Andrews \& Andrews, 2017). Other experiments reported a characteristic changes in bacteria because of different salinity conditions (Sharma et al., 2013).

\section{Effect of Inoculation on Biomass Production, Crude Protein, and Amino Acid Contents of Indigofera zollingeriana}

Despite the insignificant difference between treatments, inoculated plants could compensate the plant with nitrogen fertilizer, even better. Inoculation at $10^{5}$ $\mathrm{cfu} / \mathrm{mL}$ showed a tendency in yielding more effective biomass production and amino acid composition. Plant inoculation at higher population was assumed to disrupt the balance of carbon and nitrogen ratio. Inoculation with a higher bacteria population could 
improve nodulation activity as well as fixed nitrogen which lead to a higher accumulation of ammonium and nitrate in the soil and plant organ. Microbial activity in the formation of nodule and nitrogen fixation needs ATP as energy sources. On the other hand, ammonium and nitrate accumulation triggers transamination process. ATP synthesis and transamination need carbons. High demand of carbon caused a depletion of photosynthate. As a result, plant growth and production will not reach optimal condition.

The results showed that $I$. zollingeriana biomass inoculated with Bradyrhizobium sp. strain PZS_A08 had the highest protein content $(15.6 \%)$, although this improvement was insignificant. This result suggested that inoculation of nitrogen-fixing bacteria, such as rhizobia, effectively improved nitrogen availability, which was comparable with the use of nitrogen fertilizer in increasing leaf protein content as well as chlorophyll content (Afzal \& Bano, 2008; Namvar et al., 2013; Sara et al., 2013). The increment of chlorophyll protein content would directly affect plant production and transamination process.

Inoculation treatment with PGPR affected amino acid composition as well as its chemical score. Respectively, the addition of $\mathrm{KNO}_{3^{\prime}}$ inoculation with Bradyrhizobium sp. strain P8 828, Roseomonas sp. CMS4Y-2-2, and Bradyrhizobium sp. strain PZS_A08 increased essential and non essential amino acid composition, especially methionine and histidine. Amino acid chemical score of I. zollingeriana biomass in this experiment was higher than that of biomass with foliar treatment (Abdullah et al., 2016) and close to chemical score of top leaf flour (Palupi et al., 2016). Besides chemical score, essential and non essential amino acid composition also was significantly higher compared to previous study. However, significant difference in amino acid composition between this experiment and previous study by Abdullah et al. (2016) resulted from different planting conditions. I. zollingeriana biomass with foliar treatment was harvested after 6 month planting period and planted at a field condition (Abdullah, 2010).

Compared to human and animal, plant could synthesize 20 different amino acids (essential and non essential) through nitrate and ammonium assimilation and transamination process. Nitrate asimilation begins with a reduction process into nitrite $\left(\mathrm{NO}_{2}^{-}\right)$by nitrate reductase. Thereafter, nitrite is reduced into ammonium by nitrite reductase. Ammonium is an initial compound that is needed for syntesizing glutamine and glutamate. The availability of these amino acids determines transamination process (Taiz \& Zeiger, 2010).

Ammonium is derived from nitrate reduction, fixation process by nitrogen-fixing bacteria, and decomposition of dead bodies of living organism. Taiz \& Zeiger (2010) stated that nitrogen-fixing bacteria has nitrogenase enzyme that degrades dinitrogen triple bonds into ammonia $\left(\mathrm{NH}_{3}\right)$ which is then reacted with water and form ammonium. Available ammonium would be assimilated into glutamine and glutamate through GS-GOGAT pathway (Glutamine synthase-Glutamine: 2-oxoglutarate aminotransferase).
Synthesis of amino acid requires energy obtained from light reaction of photosynthesis and glucose catabolism. Through glucose catabolism, 3-phosphoglycerate, phospoenolpyruvate, pyruvate, 2-oxoglutarate, or oxaloacetate are produced and used for transamination. Pyruvate, 3-phosphoglycerate, and phospoenolpyruvate are derived from glycolisis, whereas 2-oxoglutarate and oxaloacetate are derived from Kreb cycle.

\section{Inoculation with Effective Strain Improves Methionine Content}

The improvemet of amino acid content such as methionine is also determined by the fitness between strain and host. In this study, methionine content of biomass with inoculation treatment was as high as methionine content in top leaf and much higher than the foliar treatment of other experiment. Other than the fact of planting periods, inoculation treatment with superior and spesific isolates was proven to be more effective in increasing methionine content.

Planting without inoculation with spesific and effective strain, in the field condition, allows random strains infecting the host. Different traits of some strains as well as the competition between strains in the soil might influence methionine content in biomass. Bradyrhizobium elkanii was found to produce rhizobitoxine which inhibited cystathionine-B-lyase in methionine biosynthesis. The inhibition increased methionine precursor and decreased methionine content in upper shoots of soybean (Okazaki et al., 2007).

\section{Methionine Improvement Related to Sulfate Assimilation}

Methionine is a limiting amino acid in leguminose plant. Inoculation of nitrogen-fixing bacteria, such as Bradyrhizobium sp. strain P8 828, Roseomonas sp. CMS4Y-2-2, and Bradyrhizobium sp. strain PZS_A08 not only enable to increase methionine percentage of the treated plant, but also increase methionine utilization. Inoculation treatment also alters the fact that methionine is a limiting amino acid. This study reveals that isoleucine is the new limiting amino acid.

Methionine is a common limiting amino acid in poultry ration other than lysine and tryptophan. If those amino acids are available in a proper amount, the utilization and availability of other amino acids will increase (McDonald et al., 2010). Bradyrhizobium sp. strain P8 828 effectively increased higher methionine content by $26 \%$ compared to methionine in I. zollingeriana top leaf (Palupi et al., 2014). Besides, it also had a high amino acid chemical score after nitrogen fertilizer treatment.

Sulfate is an essential molecule needed for nitrogen assimilation. This molecule is taken up and reduced to sulfide for the biosynthesis of nitrogenase enzyme, a complex (Fe-S) enzyme. Kalloniati et al. (2015) found that sulfate levels in root, stem, and leaves were higher than in nodules. Sulfate content in nodule was more utilized in nodules to form nitrogenase enzyme as well as other metabolites. The presence of $\mathrm{N}$-fixing nodules 
increase $S$ metabolism which improve the biosynthesis of some metabolites such as methionine, cysteine, and coenzymes.

\section{CONCLUSION}

Bradyrhizobium sp. strain PZS_A08 at $10^{5} \mathrm{cfu} /$ $\mathrm{mL}$ was the most effective strain to improve biomass production by $27.9 \%$ higher than uninoculated plant and dry matter percentage. Moreover, inoculation with Bradyrhizobium sp. strain PZS_A08 at $10^{7} \mathrm{cfu} / \mathrm{mL}$ showed the highest crude protein percentage. Bradyrhizobium sp. strain P8 828 at $10^{5} \mathrm{cfu} / \mathrm{mL}$ effectively increased amino acid chemical score as well as methionine and histidine contents. Roseomonas sp. CMS4Y-2-2 at $10^{5} \mathrm{cfu} / \mathrm{mL}$ could be also considered effective in increasing chemical score of amino acid.

\section{ACKNOWLEDGEMENT}

The funding of this study was provided by LPDP (Lembaga Penyelenggara Dana Pendidikan). Authors also thank to all who gave support to the process of experiment as well as manuscript improvement.

\section{REFERENCES}

Abdullah, L. 2010. Herbage production and quality of shrub Indigofera treated by different concentration of foliar fertiliser. Med. Pet. 33: 169-175. https://doi.org/10.5398/ medpet.2010.33.3.169

Abdullah, L., D. Apriastuti, \& T. A. P. Apdini. 2012.Use of Indigofera zollingeriana as a forage protein source in dairy goat rations. Proc. Asia dairy Goat Conference Malaysia, 71-72.

Abdullah, L., U. I. Sholehah \& N. R. Kumalasari. 2016. Kandungan Asam Amino Daun Indigofera Zollingeriana pada Beberapa Taraf Penyemprotan Pupuk Daun dan Suhu Pengeringan. Abdullah, L, D.A. Astuti, \& Suharlina, editor. IPB Press, Bogor.

Afzal, A. \& B. Asghari. 2008. Rhizobium and phosphate solubilizing bacteria improve the yield and phosphorus uptake in wheat (Triticum aestivum L.). Int. J. Agri. Biol. 10:85-88.

Alam, F., M. A. H. Bhuiyan, S. S. Alam, T. R. Waghmode, P. J. Kim \& Y. B. Lee. 2015. Effect of Rhizobium sp. BARIRGm901 inoculation on nodulation, nitrogen fixation and yield of soybean (Glycine max) genotypes in gray terrace soil. Biosci. Biotechnol. Biochem. 79:1-9. https://doi.or $\mathrm{g} / 10.1080 / 09168451.2015 .1044931$

Andrews, M. \& M. E. Andrews. 2017. Specificity in legumerhizobia symbioses. Int. J. Mol. Sci. 18:1-39. https://doi. org/10.3390/ijms18040705

Argaw, A. \& A. Tsigie. 2015. Indigenous rhizobia population influences the effectiveness of Rhizobium inoculation and need of inorganic $\mathrm{N}$ for common bean (Phaseolus vulgaris L.) production in eastern Ethiopia. Chem. Biol. Technol. Agric. 2: 1-13. https://doi.org/10.1186/s40538-015-0047-z

Bunchasak, C. 2009. Role of dietary methionine in poultry production. J. Poult. Sci. 46: 169-179. https://doi.org/10.2141/ jpsa.46.169

Cohen, M. F., X. Y. Han, \& M. Mazzola. 2004. Molecular and physiological comparison of Azospirillum spp. isolated from Rhizoctonia solani mycelia, wheat rhizosphere, and human skin wounds. Can. J. Microbiol. 50: 291-297. https:// doi.org/10.1139/w04-007.

Dé, I., K.V. Rolston, \& X.Y. Han. 2004. Clinical significance of Roseomonas species isolated from catheter and blood samples: analysis of 36 cases in patients with cancer. Clin. Infect. Dis. 38:1579-1584. https://doi.org/10.1086/420824.

McDonald, P. R. A. Edward, J. F. D. Greenhalgh, C. A. Morgan, L. A. Sinclair, R. G. Wilkinson. 2010. Animal Nutrition Seventh Edition. Pearson, Canada.

Faradillah, F., R. Mutia, \& L. Abdullah. 2015. Substitution of soybean meal with Indigofera zollingeriana top leaf meal on egg quality of Cortunix cortunix japonica. Med. Pet. 38: 192197. https://doi.org/10.5398/medpet.2015.38.3.192

Friesen, M.L. \& A. Mathias. 2009. Mixed infections may promote diversification of mutualistic symbionts: why are there ineffective rhizobia?. J. Evol. Biol. 23:323-334. https:// doi.org/10.1111/j.1420-9101.2009.01902.x

Graham, P.H. \& C.A. Parker. 1964. Diagnostic features in the characterisation of the root-nodule bacteria of legumes. Plant Soil 20:383-396. https://doi.org/10.1007/BF01373828

Kalloniati, C., P. Krompas, G. Karalias, M. K. Udvardi, H. Rennenberg, C. Herschbach, \& E. Flemetakis. 2015. Nitrogen-fixing nodules are an important sources of reduced sulfur, which triggers global changes in sulfur metabolism in Lotus japonicus. The Plant Cell 27: 2384-2400. https://doi.org/10.1105/tpc.15.00108

Kiers, E.T., R. A. Rousseau, \& R. F. Denison. 2006. Measured sanctions: legume hosts detect quantative variation in rhizobium cooperation and punish accordingly. Evol. Ecol. Res. 8: 1077-1086.

López- López, A., M. A. Rogel, E. Orme no-Orrillo, J. MartínezRomero, \& E. Martínez-Romero. 2010. Phaseolus vulgaris seed-borne endophytic community with novel bacterial species such as Rhizobium endophyticum sp. nov. Syst. Appl. Microbiol. 33: 322-327. https://doi.org/10.1016/j. syapm.2010.07.005.

Namvar, A., R. S. Sharifi, T. Khandan, \& M. J. Moghadam. 2013. Seed inoculation and inorganic nitrogen fertilization effects on some physiological and agronomical traits of chickpea (Cicer arietinum L.) in irrigated conrition. JCEA. 14: 28-40. https://doi.org/10.5513/JCEA01/14.3.1281

Okazaki, S., M. Sugawara, K. Yu-Hashi, \& K. Minawisawa. 2007. Rhizobitoxine-induced chlorosis occurs in coincidence with methionine deficiency in soybeans. Ann. Bot. 100:55-59. https://doi.org/10.1093/aob/mcm087

Palupi, R., L. Abdullah, D. A. Astuti, \& Sumiati. 2014. Potential and utilization of Indigofera sp. shoot leaf meal as soybean meal substitution in laying hen diets. JITV. 19: 210-219.

Rangin, C. G., M. Garcia, \& G. Bena. 2010. Partner choice in Medicago truncatula-Sinorhizobium symbiosis. Proc. R. Soc. B. 277: 1947-1951. https://doi.org/10.1098/rspb.2009.2072

Sara, S., M. Morad, \& C. M. Reza. 2013. Effect of seed inoculation by Rhizobium strains on chlorophyll content and protein percentage in common bean cultivars (Phaseolus vulgaris L.). Int. J. Biosci. 3: 1-8. https://doi.org/10.12692/ $\mathrm{ijb} / 3.3 .1-8$

Sharma, R.S., N.K. Rao, T.S. Gokhale, \& S. Ismail. 2013. Isolation and characterization of salt-tolerant rhizobia native to the desert soils of United Arab Emirates. Emir. J. Food Agric. 25:102-108. https://doi.org/10.9755/ejfa. v25i2.7590

Somasegaran, P. \& H. J. Hoben. 1985. The NifTAL Manual for Methods in Legume-Rhizobium Technology. University of Hawai College of Tropical Agriculture and Human Resources. US Agency for International Development.

Steel, R. G. D. \& J. H. Torrie. 1993. Prinsip dan Prosedur Statistika Suatu Pendekatan Biometrik. Gramedia Pustaka, Jakarta.

Suharlina, D.A. Astuti, Nahrowi, A. Jayanegara, \& L. Abdullah. 2016. Nutritional evaluation of dairy goat rations containing Indigofera zollingeriana by using in vitro rumen fermentation technique (RUSITEC). Int. J. Dairy Sci. 11:100-105. 
https://doi.org/10.3923/ijds.2016.100.105

Taiz, L. \& E. Zeiger. 2010. Plant Physiology. Fifth edition. Sinauer Associates Inc., publishers, Sunderland Massachusetts USA.

Tatsukami, Y \& M. Ueda. 2016. Rhizobial gibberellin negatively regulates host nodule number. Sci. Rep. 6:1-11. https://doi. org/10.1038/srep27998

Ufaz, S. \& G. Galili. 2008. Improving the content of essential amino acid in crop plants: goals and opportunities. Plant Physiol. 147: 954-961. https://doi.org/10.1104/ pp.108.118091
Wang, C, H. Y. Liu, Y. M. Wang, Z. Q. Yang, J. X. Liu, Y. M. Wu, T. Yan, \& H. W. Ye. 2010. Effect of dietary supplementation of methionine and lysine on milk production and nitrogen utilization in dairy cows. J. Dairy Sci. 93: 3661-3670. https:// doi.org/10.3168/jds.2009-2750

Zhang, J. J., T. Yu, K. Lou, P. H. Mao, E. T. Wang, W. F. Chen, \& W. X. Chen. 2014. Genotypic alteration and competitive nodulation of Mesorhizobium muleiense against exotic chickpea rhizobia in alkaline soils. Syst. Appl. Microbiol. 37: 520-524. https://doi.org/10.1016/j.syapm.2014.07.004 